\title{
Besoins des industries alimentaires : alimentation, nutrition et élaboration d'aliments
}

\author{
Gilles TRYSTRAM \\ AgroParistech, \\ ENSIA, \\ 1 Avenue des Olympiades, \\ 91744 Massy cedex \\ <gilles.trystram@agroparistech.fr>
}

Premier secteur industriel avec 145 milliards $d^{\prime}$ euros de chiffre d'affaires, l'industrie alimentaire française est aussi la première d'Europe et la seconde au monde après les États-Unis. Plus de 10000 entreprises si I'on inclut les TPE, moins de 4000 sinon, réalisent $60 \%$ du chiffre d'affaires. Ces entreprises sont des PME (90\%), quelques très grands groupes de taille mondiale qui emploient plus de 410000 personnes directement et sont encore en capacité de créer des emplois. La dernière caractéristique peu exprimée des IAA est liée au maillage économique du territoire français, car les entreprises sont implantées partout et contribuent de manière équilibrée au développement du territoire (source : www.ania.fr).

Les évolutions majeures subies et construites par le secteur sont riches, diverses et ont ces dernières années installé une évolution essentielle. La maîtrise de la sécurité alimentaire est un fait, même s'il peut rester quelques voies de progrès, il est certain que les produits sont plus sûrs qu'ils ne l'ont été et surtout que la maîtrise et l'appropriation des méthodes et des outils qui y concourt est acquise.

Étape après étape, des innovations installent un paysage des produits évoluant significativement. La recherche d'innovation pour répondre à des enjeux de marketing est constante et les salons (SIAL par exemple) divers témoignent régulièrement de la créativité des industriels, même si la déclinaison de gamme est plus la norme que l'innovation de rupture. Ces 15 dernières années, sécurité sanitaire et mâ̂trise organoleptique ont été les deux moteurs essentiels. Les enquêtes d'OSEO (www.oseo.fr) montrent régulièrement que la satisfaction du consommateur constitue l'élément déterminant des stratégies industrielles. Ainsi, I'amélioration perceptive des produits a considérablement progressé, conjointement avec praticité et adaptation au style de vie urbain notamment

\begin{abstract}
The food industries have to face new challenges and new stakes. The impact of food on nutrition and health become very important and the strategies of the companies have to change. The paper analyses the existing stakes and the way that companies have to develop in order to obtain products evolution and process reengineering. The difficulties are highlighted and the limitation of existing approaches too.
\end{abstract}

Key words: food industries, food, nutrition

(rapidité de préparation, consommation nomade).

Une nouvelle voie d'évolution s'est alors fait jour avec la recherche de satisfaction d'une demande des consommateurs de produits sains et fiables au plan nutritionnel. Cette nouvelle cible de conception de produits donne lieu à des créations, des innovations et pour certaines analyses constitue une voie pérenne de développement. II est indéniable qu'il existe conjointement un intérêt des consommateurs et un intérêt des industriels. Les uns appréciant l'idée que l'alimentation fait partie du bien être voire est capable de "soigner ", les autres $y$ voyant en plus une ouverture de marchés nouveaux, de gain de compétitivité et de développement. Un argument fort en ce sens est à trouver dans la baisse régulière de la part de budget consacré à l'alimentation pour les ménages français. Moins de $14 \%$, à comparer aux $25 \%$ des années 1970, est sans doute proche d'un minimum et sans doute les années prochaines verront remonter ce pourcentage, mais encore faut-il proposer des produits attractifs et ayant une capacité de démonstration de leur potentiel. La dimension nutrition de l'alimentation est probablement le moteur actuel de cette recherche, induisant des besoins des industriels nouveaux en matière de recherche et de développement. Mais à côté des questions liées aux consommateurs, l'évolution de la réglementation (en France et en Europe) pèse également sur le besoin de maîtrise des produits avec de nouvelles propriétés.

Dans ce contexte, à l'occasion de la journée sur I'alimentation organisée par I'Institut français de la Nutrition, il est intéressant de s'interroger sur les besoins des industriels en matière de nutrition. II ne s'agit pas de faire une enquête exhaustive mais d'extraire quelques questions parmi toutes celles qui sont posées, pour souligner les forces et les difficultés du développement de produits dans le contexte actuel. La méthode s'appuie sur les expressions publiées de ces besoins d'une part, l'expérience de l'enseignement avec notamment les élèves ingénieurs AgroParisTech, cursus ingénieur des industries alimentaires (anciennement ENSIA) et I'interrogation des services Recherche et développement de groupes industriels français. La synthèse proposée est partiale et partielle, elle tente d'extraire un point de vue et des questions, sans prétendre à l'exhaustivité. Après une analyse des enjeux actuels, trois questions sont abordées: la maîtrise des propriétés des produits et la limite des actions des industriels, la communication et ce que ces nouveaux projets induisent, la réingénierie des produits, avant de proposer une conclusion.

\section{Analyse enjeu et évolution}

De nombreux analystes dissertent sur les caractéristiques des industries alimentaires en comparaison avec les autres secteurs industriels, soulignant souvent le paradoxe entre un secteur majeur et l'absence des points forts que I'on rencontre dans les secteurs high-tech en fort développement. Dans ce cadre, il est souvent difficile et arbitraire de proposer une hiérarchie des forces et faiblesses, donc potentiellement des enjeux des industries alimentaires face à des évolutions. Un premier critère qui fait consensus est lié aux faibles marges associées aux produits. Ces marges sont reliées, outre à la capacité à payer des consommateurs, aux coûts structurels de production : frais de personnels élevés, coûts induits par la logistique pré- et postindustrielle et plus récemment l'augmentation, certainement pérenne, du coût des matières premières et de l'énergie. Une part notable des marges, contrairement à d'autres secteurs où elle est réinvestie directement en recherche et développement, est consacrée dans les industriels alimentaires à la maîtrise qualité, au respect des normes et à la 
réglementation comme aux contraintes induites par la distribution. Un enjeu majeur est donc dans la capacité d'augmenter ces marges car c'est un garant de compétitivité donc de pérennité des entreprises.

Le second enjeu majeur est lié aux contraintes que la distribution fait peser sur les producteurs $d^{\prime}$ aliments. Chercher des modes nouveaux de distribution est essentiel, notamment pour ne pas dépendre uniquement des 9 grands acteurs de la distribution et diversifier les stratégies. Les aliments répondant à des normes nutritionnelles, permettent de s'intéresser à une sectorisation plus grande et ouvrent des marchés d'interface entre alimentation et nutrition de populations à risque par exemple qui fonctionne avec des circuits diversifiés.

Le troisième enjeu notable est lié à l'évolution de la réglementation. L'alimentation se trouve du point de vue de la réglementation face à deux approches, soit l'État (France ou Europe) affirme par la réglementation le besoin de garantie nutritionnelle, soit la démarche est concertée et résulte de l'engagement contractuel des industriels. Les deux démarches existent et pèsent sur les stratégies industrielles. La notion de charte d'engagement proposée dans le cadre de Programme National Nutrition Santé 2 (PNNS2) ouvre des perspectives aux industriels ou aux groupements d'industriels, mais à condition d'avoir la capacité technologique de maîtriser ces engagements. L'évolution attendue de la réglementation vis-à-vis des allégations, la notion de profils nutritionnels, sont également un enjeu fort dont beaucoup s'inquiète en ce qu'il risque de laisser de côté les industriels n'ayant pas eu le temps ou les moyens de construire la maîtrise nécessaire.

Le troisième enjeu, indéniable si l'on en croit les enquêtes $d$ 'opinion régulières, porte sur la demande des consommateurs. Au centre des préoccupations des industriels, cette demande modifie par sa prise en compte nombre de stratégies de développement des produits. II est certain que les consommateurs demandent des produits « bons pour la santé ». Si la garantie sanitaire est un dû, largement pris en compte par les producteurs, l'expression et la traduction concrètes de ce qu'est un aliment bon pour la santé est délicate et donne lieu à des interprétations souvent contestables. II n'en est pas moins vrai que garantir des aliments ayant un rôle positif au plan nutritionnel contribuant à la qualité de la vie et au bien-être des consommateurs est un enjeu. La capacité de prévention de maladies métaboliques est de ce point de vue un enjeu certain (maladies ou pandémie comme l'obésité).

Cependant, si l'on regarde cette question à l'échelle du monde, pour le moins de l'Europe, nous sommes face à deux visions assez oppo- sées. Dans le nord de l'Europe notamment, les aliments fonctionnels sont l'essentiel de la démarche et il s'agit de proposer une vision moléculaire de l'alimentation, où les réponses aux enjeux métaboliques notamment passeront par la mise au point d'aliments fonctionnels, idéalement biodisponibles. Une autre vision, qui est sans doute davantage celle des industriels français, porte sur l'idée qu'une diète complète, ou plus de diversité, joue un rôle avant la fonctionnalité. Sans chercher à entrer dans le débat entre expert en nutrition sur ces questions, il est clair qu'un enjeu soustendu en termes de moteur au développement de produit et de leur adaptation aux questions nutritionnelles est porté par cette analyse. Les industriels français, I'ANIA notamment, défendent ces valeurs globales véhiculées par les aliments et I'alimentation. Un enjeu induit I'attractivité du secteur auprès des jeunes, futurs acteurs du secteur alimentaire.

Une approche globale de I'alimentation, incluant notamment la nutrition, la prise en compte des préférences des consommateurs, la diversité est une voie de développement où la diversité et le nombre des critères à prendre en compte interrogent significativement les démarches de développement de produits.

L'une des expressions intéressantes de ces enjeux nouveaux a été introduite en 2004 par Windhab [1]. Ce que nous avons appelé une conception raisonnée d'aliments se traduit par I'idée PAN :

- $P$ : Préférence, qui regroupe les aspects sensoriels, innovation de l'aliment ;

- A : Acceptance, qui regroupe les questions de sécurité (sanitaire, hygiène, éthique) ;

$-\mathrm{N}$ : Need, qui regroupe ce qui a trait à la santé, la nutrition, la performance.

Ce point de vue se décline de manière variée. Mais tous les auteurs actuels reconnaissent le rôle majeur du procédé et de la formulation dans cette construction globale, notamment la construction de la structure de l'aliment et son évolution en cours de traitement, voire lors de la conservation. Si l'accent est actuellement mis sur les questions de nutrition et d'impact santé au travers du critère « Need », il est possible que cette «bulle » ne va pas constituer de manière durable la voie première de création de valeur industrielle. Un constat de plus en plus partagé est que les experts en nutrition n'ont que peu de consensus sur les molécules importantes et leur environnement chimique. Les connaissances devant évoluer sans doute beaucoup dans ce domaine, la construction de méthodes et de connaissances mobilisables est plus importante que la résolution de telle ou telle question précise. Par contre, au plan des ambitions des Etats (administration, réglementation), ce sera un axe durable.
L'accent est mis aujourd'hui sur la recherche de méthodes, plus que de solutions, à même de permettre la mise au point de tailor-made food products (TMF), ou aliments "sur mesure», destinés à des demandes particulières de groupes de populations. La capacité d'intégrer des dimensions diverses dans la conception, la réalisation, la conservation et l'usage d'aliments est un axe fort, sans doute pérenne et structurant pour la recherche.

Cette construction nécessaire d'un consensus de propriétés met en particulier en avant les dimensions plaisir et bien-être associées à l'aliment. L'accent très fort mis actuellement sur la dimension santé ne doit pas faire oublier que I'interaction sensorielle avec le consommateur est un générateur premier de valeur ajoutée (qualitative et économique).

L'expression la plus structurée de ces enjeux se trouve dans le document de la plateforme Food for Life, coordonné par le CIIA (www.ciia.be), qui a fait l'objet d'un long travail d'interaction entre industriels et chercheurs. La plateforme française est en cours de finition sous l'égide de I'ANIA. Le tableau 1 présente l'extraction des enjeux et priorités issus de l'agenda stratégique résultant du travail de la plateforme Food for Life [2]. On peut y constater que les enjeux nutritionnels et donc les besoins des industriels associés à l'horizon 2020 sont distribués sur quelques-uns des challenges. Si le challenge 2 porte entièrement sur la réalisation d'une alimentation nutritionnelle, les questions de sécurité alimentaire (challenge 4) et de comportement des consommateurs (challenge 1) abordent également la question. Un point essentiel porte enfin sur la recherche de valeur ajoutée (challenge 2) et présente comme l'un des objectifs alors de concevoir des «Taylor Made Food", comme nous l'avons analysé précédemment.

\section{Qui maîtrise la chaîne de transformation?}

La transformation des aliments est l'étape-clé, car nombre d'aliments ne sont pas consommables en l'état. Le développement des industries alimentaires s'est construit sur le besoin de nourrir les populations et de retarder la consommation d'éléments nutritifs essentiels, en assurant la préservation des propriétés concernées. Le procédé vise à conserver, conférer ou inhiber des propriétés de manière durable à une matière première d'origine agricole. II module le potentiel de propriétés de la matière première et de l'éventuelle formulation associée.

Au cœur de la maîtrise industrielle se trouve la maîtrise des procédés de transformation et d'élaboration des produits. La maîtrise des pro- 
Tableau 1. Challenges et objectifs présentés par la plateforme Food for Life, Europe. Les deux derniers challenges portant sur formation et implémentation ont été ôtés.

\section{Challenge 1. Ensuring that the healthy choice is the easy choice for consumers \\ Goal 1. Measuring consumer behaviour in relation to food \\ Goal 2. Developing comprehensive models of consumer food choice processes \\ Goal 3. Promoting effective interaction with consumer groups and consumers directly through communication and public participation \\ Goal 4. Developing strategies to induce behavioural change in order to improve consumer \\ health and social responsibility (through healthier food choices)}

\section{Challenge 2. Delivering a healthy diet}

Goal 1. Understanding brain function in relation to diet

Goal 2. Understanding dietary effects on immune and intestinal function

Goal 3. Understanding the link between diet and metabolic function (obesity and associated

metabolic disorders)

Goal 4. Understanding consumer behaviour in relation to health and nutrition

Challenge 3. Developing value-added food products with superior quality, convenience, availability and affordability

\section{Goal 1. Producing tailor-made food products}

Goal 2. Improving process- and packaging design and process control

Goal 3. Improving understanding of process-structure-property relationships

Goal 4. Understanding consumer behaviour in relation to food quality and manufacturing

\section{Challenge 4. Assuring safe foods that consumers can trust}

Goal 1. Predicting and monitoring the behaviour and fate of relevant known and emerging biological hazards.

Goal 2. Predicting and monitoring the behaviour and fate of relevant known and emerging

chemical hazards including toxins of biological origin

Goal 3. Improving risk assessment and risk-benefit evaluation

Goal 4. Developing tools to ensure security of the food chain

Goal 5. Understanding and addressing consumer concerns with food safety issues

\section{Challenge 5. Achieving sustainable food production}

Goal 1. Understanding the sustainability of food production and supply in Europe

Goal 2. Developing scenarios of future European food production and supply

Goal 3. Developing sustainable processing, packaging and distribution

Goal 4. Developing and implementing sustainable primary food production

Goal 5. Understanding consumers and their behaviour regarding sustainable food

production

\section{Challenge 6. Managing the food chain}

Goal 1. Identification of possible scenarios

Goal 2. Stabilising markets and supporting food chain dynamics through the generation and

preservation of trustGoal 3. Improving the innovation potential of the food chain

Goal 4. Supporting competitiveness through integration

Goal 5. Participation of small producers in complex food chain operations

Goal 6. Integrating food chain management and the consumer

cédés industriels a certes considérablement progressé, mais il reste de la méconnaissance. En particulier, si durant de nombreuses années les principes d'action physique, chimique et biologique sur les ingrédients ont été analysés, ce sont surtout les critères sensoriels ou technologiques (aptitude au transport, à la découpe, etc.) qui ont été étudiés. La dimension nutritionnelle et chimique plus généralement est peu développée. Cela laisse des zones d'ombre importantes sur les mécanismes d'action, et donc limite la capacité de maîtrise. L'intérêt autour de la composition nutritionnelle des aliments génère des études nouvelles enrichissant les connaissances, mais nous ne sommes qu'au début de ces actions. On peut par exemple noter que nombre des actions de recherche du Programme National Recherche Alimentation (PNRA) cadre d'un appel d'offre de l'ANR ces trois dernières années a porté sur ce type d'analyse. L'obtention de résultats devrait aider à progresser.
Le diagnostic du potentiel des opérations de transformations est essentiel à maîtriser. Des actions de recherche récentes ont été entreprises pour construire des outils allant en ce sens. Une formalisation assez complète des démarches est disponible dans un ouvrage coordonné par I'ACTIA [3]. Dans ces démarches, une des limites est le coût des analyses dès lors que I'on s'intéresse aux compositions chimiques des aliments, voire à l'analyse clinique des impacts nutritionnels pouvant se concrétiser en vraie allégation démontrée.

Mais croire que la seule maîtrise de l'étape industrielle suffit est illusoire. L'industrie alimentaire n'est que le premier maillon de la chaîne de l'alimentation. Certes essentiel en termes de conservation de propriétés, ce maillon construit des propriétés que les étapes successives peuvent annuler. La conservation est une étape clé et le devenir de composé $d^{\prime}$ 'intérêt nutritionnel est encore faible lors du processus de conservation. La difficulté de certaines des études est grande, en particulier pour les produits à long terme de conservation.

L'étape suivante est liée à l'usage de l'aliment. À ce titre beaucoup de questions interrogent les producteurs d'aliments. En effet, au plan nutritionnel, si l'on comprend que le processus de transformation, souvent thermique, influence la composition et la biodisponibilité, I'usage et la manière dont le produit est cuit, réchauffé, voire conservé peut modifier ces propriétés installées par le transformateur. Cette étape, qu'elle se situe à un stade d'usage domestique ou d'usage en restauration hors foyers (RHF), est essentielle et mal maîtrisée. II existe actuellement peu d'études disponibles et cette méconnaissance pèse sur les stratégies de développement de produits.

II existe un consensus sur le fait que la formation des consommateurs est une étape essentielle à la maîtrise des propriétés d'intérêt nutritionnel des aliments. Du reste, on peut noter que dans le cadre du PNNS 2 et en particulier des chartes d'engagement nutritionnel, des actions vers le consommateur et vers les employés des usines ou entreprises de transformation d'aliments sont recevables. Les entretiens avec les industriels montrent bien que cette dimension est très intégrée dans les démarches et constitue un levier d'action significatif. Ce point souligne l'importance de la dimension de communication sur ces questions d'impact nutritionnel des aliments. On peut s'interroger sur le fait de savoir si la diversité de la communication qui est proposée est vraiment informative pour le consommateur. 


\section{Démarches pour répondre aux enjeux : réingénierie des aliments et des procédés}

Les progrès successifs des industries alimentaires ont consisté en l'amélioration étape après étape en incluant un critère nouveau à chaque fois dans le réglage soit de la formule soit du procédé. II arrive un temps où cette approche est trop contrainte. Le fait que des ingrédients aient été mis en œuvre par exemple avec des vertus technologiques (garantie de productivité, robustesse du procédé par exemple) ou que l'incrément organoleptique ait été fait est indéniable. Mais dès lors que l'on complique la cible du produit en apportant d'autres propriétés, par exemple nutritionnelles, les études deviennent plus complexes et les compromis acquis sont largement remis en cause. La seule voie d'approche mobilisable est d'assurer une réingénierie du procédé et des formulations.

Un certain nombre d'industriels ont déjà engagé une vision d'ensemble consistant à redéfinir l'ensemble des recettes et les réglages associés des procédés. II s'agit d'une vision nécessairement nouvelle consistant à revisiter totalement la manière de produire un aliment. L'analyse est délicate pour en extraire une méthode globale, mais un certain nombre de points sont d'ores et déjà à souligner.

Paradoxalement, le poids de l'appropriation interne est important. Cet aspect en général sous-estimé est essentiel, car les premiers à être mobilisés pour améliorer ou redéfinir les aliments et leur processus de transformation sont les employés des transformateurs. Expliquer, convaincre, justifier en interne apparaît une étape essentielle. L'observation de quelquesunes des premières chartes d'engagement nutritionnelle montre ainsi que cette dimension est prise en compte et que la formation à la nutrition interne aux entreprises est de première importance. Cet aspect n'est pas souvent considéré, mais l'expérience montre son intérêt.

Dans la réingénierie, la manière de penser doit changer. Les sciences de l'aliment, le génie des procédés ont construit des méthodes et des outils au fil des années qui consistent essentiellement à tester des situations, sur une base grandement expérimentale, à constater des effets, les raisonner et ensuite en déduire des voies d'améliorations. Souvent un constat résultant est une idée d'innovation ou d'amélioration des produits, notamment vers de nouvelles propriétés organoleptiques. En matière nutritionnelle, la question se pose et s'aborde différemment. En effet, le produit doit être conforme au plan organoleptique avant tout. II faut donc partir de la cible, recenser les besoins et construire des réponses à ces besoins. L'expression de l'ensemble des propriétés à

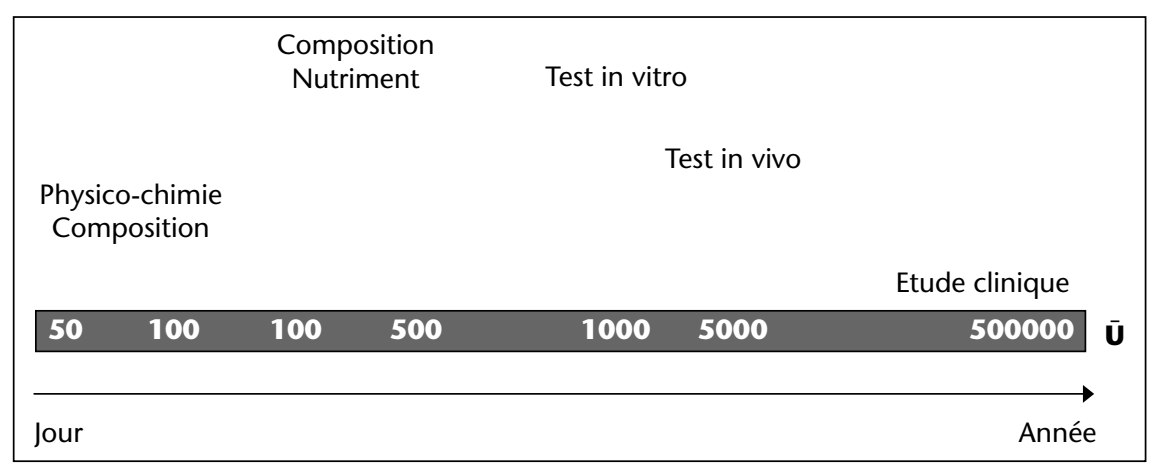

Figure 1. Échelle temporelle et économique associée aux études en matières de construction de propriétés nutritionnelles validées lors de la transformation d'aliments.

construire et la recherche de toutes les voies technologiques qui y concourent constituent alors la démarche appropriée. II existe peu de méthodes validées explicitement pour ces démarches, mais il est clair et il y a consensus sur l'idée que c'est dans ce sens que doit se construire l'approche nouvelle.

Ces approches doivent alors prendre en compte une bonne définition de la cible nutritionnelle considérée. II se situe là une difficulté. Prendre en compte une recommandation générique de type PNNS2 sur le sel ou le sucre ajouté ou sur la nature des matières grasses est faisable. II existe dans certains cas des limites technologiques nécessitant des travaux importants, mais c'est réalisable. Dès lors que l'on $s^{\prime}$ intéresse aux molécules justifiant des revendications d'intérêt, la situation est plus complexe. Pour le moins deux raisons sont à mettre en exergue.

La première raison est liée au fait que la littérature ne fait pas consensus au plan nutritionnel. II existe certes des données reconnues, mais aussi des résultats publiés contradictoires quant aux effets de certaines molécules. Manquant d'outils aptes à fournir des réponses en termes de biodisponibilité effective, manquant aussi de connaissances sur les effets d'interactions entre molécules, la prise de recul sur les données publiées et complexes. Les avis d'experts divergent parfois, et donc la position des choix industriels en est compliquée.

La seconde raison est celle du coût des études. La figure 1 illustre la double échelle temporelle et économique face à laquelle les industriels sont confrontés. D'une part, les coûts des analyses impactant les décisions en termes nutritionnels se situent sur une échelle considérable jusqu'aux études cliniques qui sont très coûteuses. D'autre part, comme l'illustre la figure, l'échelle de temps pour obtenir un résultat dépasse souvent l'échelle de décision normale des industriels qui pour les PME notamment est rarement au-delà de l'année. Derrière les données portées sur la figure se trouvent bien sûr des situations très diverses, selon les molécules, les matrices et les connaissances antérieures en matière de chimie des aliments ou de nutrition. Cependant cette figure illustre aussi le facteur de risque fort que des PME n'aient pas les moyens économiques d'assumer les études nécessaires.

\section{Conclusion}

La diversité des besoins des industries alimentaires est grande. La prise en compte d'une dimension nutritionnelle des produits et les conséquences vers l'ouverture de nouveaux marchés constituent des enjeux forts et des moteurs importants du développement. Les approches classiques ne sont pas nécessairement appropriées et la rénovation des produits et des procédés demande de nouveaux outils. Tous les secteurs industriels ne sont pas à égalité dans cette situation, notamment entre grands groupes, PME et TPE, les possibilités ne sont pas identiques. Au sein des PME, la situation financière ne permet pas toujours d'embaucher de cadre dédié à la recherche et développement. La capacité d'investissement en recherche et développement (moins de $0,5 \%$ du chiffre d'affaires) n'est pas toujours en lien avec les besoins induits par une compétition lourde sur le champ de la maîtrise des propriétés nutritionnelles. Néanmoins, si l'on considère le placement $d$ 'ingénieurs $d^{\prime}$ une formation comme celle d'AgroParisTech, cursus des industries alimentaires, $30 \%$ des emplois sont en recherche et développement; ce qui montre aussi la nature du besoin dans l'industrie, mais aussi la dynamique qui existe pour innover toujours plus.

La complexité des aliments transformés, générés notamment par les attentes du marché, ne cesse d'augmenter. Les industriels doivent faire face à de nouvelles exigences de qualité (nutrition, praticité) et de nouvelles contraintes (environnement, traçabilité, etc.) imposées soit par la réglementation, soit par leurs clients. 
Certaines grandes entreprises les maîtrisent en partie. Pour les PME, le défi devient de plus en plus difficile.

Pour tous les secteurs des industries alimentaires, il est probable que les prochaines années vont changer considérablement tant les procédés que les formulations. C'est un impact direct et indirect d'une préoccupation de maîtrise des propriétés nutritionnelles, sanitaires, organoleptiques et technologiques des aliments.

\section{RÉFÉRENCES}

1. WINDHAB E, 2004, ICEF9 CD Rom, Édition SCI, 28 rue saint dominique, 75008 Paris.
2. Stakeholders' Proposal for a Strategic Research Agenda 2006-2020, CllA, plateforme food for Life, Mai 2007, http://www.etp.ciia.be.

3. ACTIA, 2005, Guide audit nutritionnel. Edition ACTIA, 16 rue Claude Bernard, 75231 Paris, cedex. 